\title{
PROCESOS DE INDUSTRIALIZACIÓN DE LOS MEDIOS DE PRODUCCION MARINOS EN LAS COSTAS DEL NORTE DE CHILE (1935-1938). EL CASO DEL PLAN PESQUERO DE TARAPACÁ*
}

\author{
INDUSTRIALIZATION PROCESSES OF MARINE PRODUCTION \\ MEDIA ON THE NORTHERN COASTS OF CHILE (1935-1938). \\ THE CASE OF THE FISHING PLAN IN TARAPACÁ
}

\author{
CLAUDIO AGUIRRE**, CARLOS MONDACA***
}

Resumen: En este artículo se analiza el rol del Instituto de Desarrollo de Tarapacá y su Plan de Pesca en la conformación de las industrias pesqueras en las costas del norte de Chile. Se infiere desde el análisis de fuentes históricas de la época y contemporáneas, que este proceso fue posible de implementar debido a la situación de crisis que se vivía a causa de la crisis global del capitalismo iniciada en 1929 y al declive de la industria del nitrato en Tarapacá. En este contexto, se sostiene que la actividad pesquera industrial, los recursos marinos y los medios de producción haliéuticos fueron prioridades para la nueva lógica económica dirigida desde el Estado, y que estos elementos fueron un polo de desarrollo concreto que potenció la importancia estratégica del territorio marítimo y de su soberanía nacional, abriéndose un mercado tanto nacional como internacional al capitalismo industrial en la pesca.

PAlabras Clave: Industria pesquera, Instituto de Fomento Pesquero de Tarapacá, Plan pesquero de Tarapacá, norte de Chile, recursos haliéuticos.

Abstract: This article analyzes the role of the "Instituto de Desarrollo de Tarapacá" and its Fishing Plan in the conformation of the fishing industries in the coasts of northern Chile. It is inferred from the analysis of historical sources of the time and contemporary sources, that this process was possible to implement due to the critical situation brought about by the global crisis of capitalism that began in 1929 and the decline of the nitrate industry in Tarapacá. In this context, it is argued that industrial fishing activity, marine resources and halieutic means of production were priorities for the new economic logic directed by the State. And that these elements were a concrete pole of development that enhanced the strategic importance of the maritime territory and

\footnotetext{
* Este trabajo es parte del Proyecto Fondecyt de iniciación No 11190353, y de Investigación Mayor de Ciencia y Tecnología UTA Mayor 5727-20.

** Doctor en Antropología. Académico del Departamento de Educación, Universidad de Tarapacá, sede Iquique, Iquique, Chile. Correo electrónico: caguirre@uta.cl. Orcid: https://orcid.org/0000-00017378-2507

*** Doctor en Educación. Académico del Departamento de Educación, Universidad de Tarapacá, Arica, Chile. Correo electrónico: cemondacar@uta.cl. Orcid: http://orcid.org/0000-0003-2640-2705
} 
its national sovereignty, opening up a national and international market for industrial capitalism in fishing.

Keywords: Fishing Industry in Chile, Fishing Development Institute of Tarapacá, Tarapacá Fisheries Plan, North of Chile, Halieutical Resources.

Recibido: 22.08.2019. Aceptado: 11.05.2020.

\section{INTRODUCCIÓN}

T A CRISIS GLOBAL del capitalismo de 1929 y el paulatino ocaso de la inLdustria salitrera en el norte de Chile, propiciaron profundas transformaciones de las políticas económicas de los gobiernos de la época, las cuales impulsaron la industrialización y diversificación productiva nacional bajo el modelo del desarrollo hacia adentro y de sustitución de importaciones. En esta nueva visión de desarrollo, los recursos marinos de las costas chilenas fueron considerados como elementos productivos posibles de ser industrializados. Para el caso de las costas tarapaqueñas estas posibilidades se acrecentaron en forma vertiginosa, dada la precaria situación social y económica en la cual se encontraba la región y especialmente la ciudad de Iquique producto de la crisis en la industria salitrera (Aguirre, 2016).

Para poner en marcha los planes de despegue económico de las regiones del norte de Chile, se crearon bajo Ley No 5.546 de 1934 los institutos de fomento industrial del norte, como instituciones autónomas, pero dependientes del Ministerio de Fomento de la época, con sedes en Iquique y Antofagasta. Dichas instituciones tenían como objetivo elaborar planes de fomento industrial minero, agrícola y pesquero. A mediados de 1935 se pone en funcionamiento el Instituto de Fomento de Tarapacá, el cual rápidamente plantea como una de las primeras medidas de resurgimiento económico un Plan Pesquero en 1936, que se materializa a partir de la formación de una sociedad anónima conocida como "Consorcio Pesquero de Tarapacá, mediante el Decreto con Fuerza de Ley No 251 de Marzo de 1931. Dicha entidad tenía como objetivo la planificación y construcción de toda una infraestructura y organización industrial pesquera que incluía frigoríficos, fábricas de conservas, harinas y aceites de pescado, construcción y adquisición de embarcaciones y de artes "modernas" de pesca, planteando al mismo tiempo la construcción y compra de viviendas para los pescadores y sus familias. Bajo este contexto, podemos observar los primeros in- 
tentos estatales por planificar, organizar, promover, financiar y modernizar los medios de producción marinos de las costas nortinas.

En este marco, el objetivo de este artículo es analizar los primeros pasos del proceso de industrialización pesquera que está centrado en el discurso desarrollista que guía dicho modelo económico, el cual vio en las condiciones económicas y sociales imperantes en la década de los años treinta del siglo pasado una fuente de plausibilidad para sus postulados, lo que se manifestó por parte del Estado chileno en la generación de políticas económicas y sociales que estimularon la inserción y desarrollo del capitalismo industrial en la pesca.

Nuestra hipótesis de trabajo sostiene que este proceso fue posible de implementar debido a la emergencia económica que vivía un importante número de la población de la región, escenario que influye significativamente en la proliferación de discursos industrializadores dirigidos a los medios de producción haliéuticos (Bolster, W. J., 2006). Estos discursos van acompañados de distintas medidas económicas y sociales que buscan generar las condiciones para la apropiación, capitalización y modernización de los procesos productivos pesqueros. De esta forma, cuando el Estado chileno ve en la actividad pesquera industrial una posibilidad de desarrollo, se potencia la importancia estratégica del territorio marítimo y de su soberanía nacional, abriéndose un mercado tanto nacional como internacional al capitalismo industrial en la pesca, proceso que también se repite en otros países latinoamericanos con recursos marinos y extensas costas.

Esta investigación utilizó una metodología cualitativa, siendo la principal estrategia de investigación el análisis histórico documental, centrado especialmente en recolectar y analizar información proveniente del plan pesquero regional para el período 1936-1938, así como en otras fuentes documentales y bibliográficas. Toda la información recolectada fue triangulada y luego analizada utilizando la técnica de análisis crítico de contenido.

\section{LA INTERVENCIÓN JURÍDICA DEL ESTADO CHILENO EN LA INDUSTRIA PESQUERA}

Existieron en el pasado iniciativas industrializadoras a partir de la Ley de Pesca de 1906 (Albert, 1913), y que de acuerdo a Camus et al. (2016) con estas se fueron plasmando una serie de medidas industrializadoras en las costas del sur de Chile, las cuales apuntaban al control de los recursos marinos, 
a partir de la concentración de los medios de producción, la proletarización intensiva de los productores, la dependencia del capital a través de créditos, el control de los flujos de comercialización y los mecanismos de control de la fuerza de trabajo, donde el Estado ha jugado un papel importante en la promoción de leyes que regulan la actividad, que se visualizan en las diferentes legislaciones pesqueras (1912, 1916, 1918 y 1919).

Aun así, gran parte de estas iniciativas fracasó, ya que se contaba con escasa tecnología, y existía un bajo desarrollo de conocimiento científico y técnico para su explotación industrial, además de las respuestas de los pescadores que se resistían a la innovación y a los cambios del proceso en marcha (Durrenberg y Pálsson, 1987; Aguirre, 2011; Aguirre et al., 2014).

De igual manera, Harms (1930) argumentó para ese proceso inicial la falta de apoyo estatal y la nula respuesta de los capitalistas, lo cual frustró unos de los proyectos emblemáticos de la época: la "Compañía Pesquera Estrella de Chile", que se proyectaba instalar en la Bahía de Quinteros, próxima a Valparaíso. La ausencia de ayuda estatal y el poco interés en invertir de parte de sectores capitalistas en un espacio productivo desconocido, fueron elementos claves que retrasaron la vertiente industrial en las costas nacionales.

Por lo tanto, no es hasta el momento de la crisis económica en el norte que comienzan a surgir discursos que abogan por organizar la industria pesquera como ocurre en otros países mediante una intervención activa y planificada por parte del Estado, aunando esfuerzos con sectores privados, con la finalidad de iniciar el desarrollo de una industria pesquera. La siguiente cita describe lo planteado: "No cesaremos de repetir que la industria de la pesquería, en nuestro país debe merecer especiales deferencias de parte del Estado, por estar tan estrechamente vinculada al problema de la subsistencia" (Harms, 1930, p. 113).

En 1931 el Estado comienza a intervenir jurídicamente a través de la aprobación de la Ley de Pesca de ese año, la cual es publicada en 1934. Dicho cuerpo legal establece las condiciones para la regulación y control de la pesca y los productores, mediante la organización de los pescadores y sus medios de trabajo, la promoción de la educación para el consumo de pescado y, por último, la creación por parte de instituciones públicas de instalaciones que permitan la distribución de la producción pesquera (Orrego, 1973). Si bien el Estado procura las condiciones para la implementación de esta industria, esta solo se manifiesta en la administración del espacio y su población, sin una inversión efectiva en el sector pesquero. De acuerdo a estas bases jurídicas, se instalan en el puerto de Iquique las primeras indus- 
trias pesqueras de capitales privados pertenecientes a las firmas Lauder y Korps $^{1}$, industrias dedicadas a la pesca y distribución de pescado fresco y en conserva ${ }^{2}$, estando la mayor parte de la producción destinada al mercado local y nacional.

En este contexto, se puede sostener que estas empresas fueron las primeras iniciativas industrializadoras en los espacios marinos tarapaqueños, sin embargo, estas unidades productivas presentaban los problemas clásicos de planificación y organización de esta actividad extractiva, dadas por la especificidad ecológica del ambiente marítimo, que se caracteriza por lo inestable de las condiciones del clima y la movilidad migratoria de los peces, lo cual hace que sea muy difícil predecir el aumento o disminución del recurso marino. Junto con ello, se debe agregar la dificultad de apropiación de los medios de producción marinos y la imposibilidad aparente de un control sobre los productores y de sus actividades en el mar.

Estas dinámicas ecológicas y socioeconómicas dificultan la planificación en este tipo de industria, que inciden tanto en la inversión como en las posibles ganancias, afectando el proceso de acumulación de capital en este tipo de actividad económica. En atención a esta realidad, las fábricas de esta industria pesquera que se instalaron en Iquique, solo se dedicaron al proceso de elaboración y comercialización del producto marino, dejando la organización extractiva a los pescadores. Al respecto, tal como lo plantea Alfaro (1936), los pescadores se procuraban la provisión de los materiales de pesca por sus propios medios, sin ninguna ayuda de terceros.

En ese sentido, los riesgos de la pesca se trasladaban a los productores (McCay, 1978), donde existe la imposibilidad de realizar un proceso de acumulación en medios de producción (embarcaciones y artes), debido al carácter fungible de estos, lo que obliga al capital a concentrar sus objetivos en otros medios para la realización de esa acumulación alejándose del proceso extractivo (Alegret, 1988, p. 21).

Esta situación influyó en las estrategias que desplegó el capital para poder tener un control efectivo de las fuerzas de trabajo y, por ende, retrasó el cumplimiento de sus objetivos de acumulación y valorización del capital producto de la escasa tecnologización que se registró en las primeras épocas del capitalismo mercantil debido a la abundancia del recurso, como

\footnotetext{
${ }^{1}$ De acuerdo al artículo 8 de la Ley de Pesca de 1931, se podía conceder por arrendamiento porciones de playas para la instalación de industrias destinadas a las labores pesqueras.

2 Tales fábricas tenían entonces maquinarias para la confección de los envases y contaban con considerables partidas de hojalatas traídas desde el extranjero (Alfaro, 1936, p. 255).
} 
también a la dificultad de control de la fuerza de trabajo, lo que empujó al capital a dedicarse exclusivamente a la comercialización de la producción en un momento dado (Durrenberger \& Pálsson, 1985; Alegret, 1988; Breton y López, 1989). No es hasta la intervención del Estado, ahora en términos económicos, que estas dificultades tratarán de ser resueltas.

\section{PLAN PESQUERO DEL INSTITUTO DE FOMENTO DE TARAPACÁ Y APROPIACIÓN DE LOS MEDIOS DE PRODUCCIÓN MARINOS POR PARTE DEL ESTADO CHILENO}

En 1935 se comienza a concretar la intervención económica del Estado en la pesca por medio de agencias locales de desarrollo, haciéndose eco de los llamados de distintos sectores a intervenir y controlar los nuevos espacios productivos. En virtud de ello se planteaba que debía existir una amplia libertad industrial y un oportuno fomento a favor de la actividad pesquera en su sentido más amplio, porque era una fuente de riqueza nacional, además de un valioso medio de alimentación popular (Instituto de Fomento Pesquero de Tarapacá, 1936, p. 1285). Es evidente que sobre estos fundamentos la industrialización pesquera tendrá su justificación como única alternativa viable de desarrollo y progreso y, como lo plantea Lander (2002), estaríamos frente a un proceso de expansión de la lógica de mercado en espacios que no habían estado plenamente sometidos. De esta manera, se comienza a vislumbrar el rol que jugará el Estado en la planificación y control de los medios productivos marinos a partir del Plan de Fomento Pesquero, el cual comienza a materializarse con la instalación de la primera industria pesquera con capitales mayoritariamente estatales en el puerto de Iquique.

Dicha pesquera será conocida como "Cavancha", la cual se instala en los espacios de una antigua caleta de pescadores del mismo nombre en Iquique. En 1936 se presentan las principales medidas inmediatas del Plan

\footnotetext{
${ }^{3}$ Este plan se fundamentó principalmente en dos investigaciones científicas realizadas en el país. A saber, investigaciones ictiológicas desarrolladas por el doctor alemán Hans Lübbert contratado por el gobierno chileno en 1926, que tras sus viajes por las costas chilenas, emitió un informe sobre las posibilidades de industrialización de los medios de producción marinos en el litoral nacional, planteando además la inversión de recursos financieros para la modernización de las artes de pesca (Aguirre et al., 2018). También encontramos los estudios sobre el estado de la nutrición en el país, los cuales fueron realizados por los profesores Carlos Dragoni y Etienne Burnet, peritos de la Sociedad de las Naciones, los cuales valoraron el aporte nutritivo de los productos marinos como fuente alimenticia para los sectores desposeídos (Instituto de Fomento Pesquero de Tarapacá, 1936).
} 
Pesquero, como es la construcción de un puerto pesquero acorde a los fines industriales, la instalación de un frigorífico para la conservación de la pesca, el mejoramiento del transporte del producto, la educación industrial pesquera y la propaganda para incentivar el consumo de pescado. En cuanto a las artes de pesca se indica la necesidad de modernizar los procesos de captura a partir de la construcción y motorización de las embarcaciones para ser entregadas a los pescadores a través de créditos estatales. Para 1937 el Plan Pesquero se materializa con la puesta en marcha de toda una infraestructura productiva en las costas nortinas ${ }^{4}$. Dicha infraestructura estaba destinada a generar las condiciones previas que permitirán la realización de procesos de acumulación de capital y, por consiguiente, acceder a la propiedad de los medios de producción (Alegret, 1988).

Si bien el Plan Pesquero había concretado inicialmente gran parte de lo planificado, se estaba consciente de las dificultades de mantener y expandir este tipo de industria. Esto se grafica en la siguiente cita: "Emprender el problema en esta forma integral requeriría la disposición de cuantiosos capitales, y aún, suponiendo que se contara con los recursos financieros necesarios, el desarrollo de la industria requiere varios años de plazo" (Instituto de Fomento Pesquero de Tarapacá, 1936, p. 64).

No obstante, la necesidad de generar rápidamente una alta productividad en este sector llevó a elevar la composición orgánica del capital como forma de controlar el objeto de trabajo y los productores. De acuerdo a ello: “(...) desde sus primeras sesiones, el Consejo del Instituto de Tarapacá se preocupó preferentemente del Crédito Pesquero, con la finalidad inmediata de hacer resurgir la industria pesquera en la provincia (...)" (Instituto de Fomento Pesquero de Tarapacá, 1936, p. 60). Estos créditos consistían en la entrega de herramientas y materiales, tales como: embarcaciones, redes, combustible, repuestos para motores y toda clase de útiles necesarios para las faenas en el mar. De esta forma la industria se aseguraba la modernización de las artes de pesca y el control de la fuerza de trabajo mediante la práctica de contratos y, a su vez, se cercioraban del surtimiento de pescado mediante el mismo mecanismo.

La inversión en medios de producción en estos espacios es considerablemente alta, por lo tanto, elevar la composición orgánica del capital, como forma de controlar, no asegura una alta productividad de acuerdo

\footnotetext{
${ }^{4}$ Muelle, frigorífico, pabellones para el secado y salazón del pescado, astillero y la línea de conserva. Asimismo, se implementó la enseñanza industrial conforme a las necesidades de la industria. Junto con ello se planificó la modernización de la distribución y comercialización de los productos, entre los mercados del Puerto de Iquique y la pampa, a partir de la compra de camiones frigoríficos.
} 
a lo invertido. Como sabemos, la planificación productiva de este tipo de industria requiere plazos mayores, producto de las dificultades antes nombradas. Dichos obstáculos comenzaron a hacerse presentes en este proceso, como es consignado en la siguiente cita:

Nunca les faltó a los pescadores que trabajan en las embarcaciones del instituto, el petróleo o gasolina necesarios, sus redes, elementos varios de pesca. Aunque la pesca de uno o varios viajes fuera escasa jamás faltaban los elementos para salir nuevamente. Ahora por razones de incomprensión sobre las finalidades pesqueras del instituto, la directiva y consejo del instituto se vio precisada a renunciar a su control riguroso y directo sobre las faenas pesqueras de sus embarcaciones. (Instituto de Fomento Pesquero de Tarapacá, 1936, p. 66)

La argumentación recién planteada permite comprender las diferencias entre los procesos productivos pesqueros y otros procesos como la agricultura y la minería, puesto que no es fácil arrendar ni vender el acceso al mar y sus recursos, por la materialidad de este y la movilidad y dispersión del recurso mismo. A pesar de los esfuerzos por controlar los procesos productivos marinos, la actividad extractiva en este período seguía incompleta.

Según CORFO (1967), había un desconocimiento de las características de los recursos marinos (abundancia, distribución en espacio y tiempo y variaciones de la disponibilidad), especialmente de las especies que tienen una mayor importancia económica, lo que generaba incertidumbres al momento de iniciar cualquier tipo de inversión mayor en el sector. De manera análoga, CORFO (1967) señaló que la carencia de recursos de las pesquerías chilenas antes de 1959 estaría dada por el escaso desarrollo tecnológico que permitiera implementar una industria de este tipo, ya que casi la totalidad de las embarcaciones anteriores a 1959 eran bongos o chalupas escasamente motorizadas e incluso con viejos motores de automóviles. De acuerdo con ello, debieron pasar treinta años para que se dieran las condiciones necesarias para la masificación del capitalismo industrial en estas latitudes. Según Aguirre et al. (2018), a partir de 1939, con la aplicación del Plan Nacional Pesquero de la CORFO, comienza una nueva etapa en el desarrollo de las pesquerías nacionales, con la contratación de nuevos estudios científicos en el extranjero, como es el caso de la agencia gubernamental de Estados Unidos: The United States Fischeries and Wildlife Service (USFWS) en 1943.

A ello habría que agregar la aparición de un mercado global a finales de la década de los cincuenta, que se manifestó en una creciente demanda de 
proteínas de origen marino para satisfacer la necesidad de la alimentación de los animales de corral, la sobreexplotación de los recursos marinos de la costa californiana y las primeras medidas proteccionistas ambientales en los países del norte. Todo ello provocó que la inversión y el capital asociados a la industria pesquera desarrollada en el hemisferio norte se expandieran hacia las costas del Pacífico Sur, cambiando para siempre la historia pesquera del extremo norte de Chile.

\section{EL PLAN PESQUERO Y LOS PESCADORES: GOBERNABILIDAD EN LAS COSTAS DE TARAPACÁ}

El agente principal dentro de la estructura económica de las pesquerías es la fuerza de trabajo, siendo los productores el engranaje clave en la conformación económica del sector. De esta manera, los esfuerzos del capital por la apropiación de los medios productivos marinos tendrán como primer objetivo ordenar, disciplinar, controlar y regular la fuerza de trabajo, con el fin de asegurar y proveer un contingente laboral adecuado a los fines del modo de producción industrial. Dentro de este proceso de control y disciplinamiento social nos encontramos con el dispositivo jurídico plasmado en la Ley de Pesca de 1931, que comienza a configurar la relación entre los pescadores y el Estado, a partir de la regulación a la libertad de acceso a las playas ${ }^{5}$ y el ejercicio de su trabajo a toda persona que ejerza la profesión de pescador, y establece el control y la decisión de expulsar a los pescadores que no cumplan los requisitos impuestos en la Ley en su Art. 89: "Los sitios que formen parte de una población pesquera serán concedidos por plazos indefinidos, y el Gobierno podrá cancelar la ocupación en cualquier momento a aquel o aquellos pescadores que dejen de reunir las condiciones exigidas" (como se citó en Orrego, 1973, p. 233). Esta normativa buscaba en primer lugar reafirmar la apropiación por parte del Estado, de un espacio considerado común y de libre acceso. También nos encontramos con las primeras señales de regulación de un mercado de trabajo, lo que significa un control de una población específica: los pescadores. En consecuencia, estamos frente al concepto de la razón gubernamental que enuncia Foucault (2001) como principio estructurador de las relaciones del Estado con las poblaciones pesqueras y el mercado.

\footnotetext{
${ }^{5}$ Toda persona que ejerza la profesión de pescador deberá tener su carnet de identidad e inscribirse en los registros especiales de la Gobernación Marítima o departamental respectiva.
} 
Lo anterior nos permite entender de mejor forma las medidas posteriores adoptadas por el Plan Pesquero en las poblaciones costeras del litoral nortino, las cuales van a tono con la nueva mirada que se tiene de los pescadores como objeto y sujeto de una economía política. En este sentido, las primeras disposiciones adoptadas sobre las poblaciones pesqueras estaban dirigidas hacia la vigilancia y control de estas, a partir de un plan de radicación y concentración de los habitantes en villas aledañas a las nuevas fábricas. Junto con ello, se implanta la prohibición del consumo de alcohol dentro de estos lugares.

Estas medidas restrictivas se justificarían a partir de los discursos eugenésicos que planteaban la incivilizada forma de vida de estos sujetos, ajenos a todo beneficio previsional y fuera del engranaje económico nacional, lo que hace que sus actividades no reporten una real utilidad a la sociedad en que viven (Mendoza, 1962).

Estas características sociales y culturales eran consideradas inadecuadas, improductivas, peligrosas y opuestas al principio racional del cálculo contable del modo de producción industrial. De esta manera, de acuerdo al Plan Pesquero, se ideó mejorar las condiciones sociales y económicas de los gremios del sector. Este planteaba que:

(...) un hogar limpio y riente, lleno de luz y de sol, sin parásitos ni emanaciones fétidas, es una garantía de felicidad para los esposos, de salud para sus hijos y de moralidad. El hogar sucio y repulsivo, es el veneno más fatal para la dicha conyugal, la muerte prematura de los nenes (...). (Instituto de Fomento Pesquero de Tarapacá, 1936, p. 665)

En el mismo tenor se argumentaba que se debía:

(...) velar por la salud y moralidad de la gente dedicada a la pesca y defender el hogar contra el alcoholismo, para lo cual el instituto obtuvo del supremo gobierno la declaración de zona seca dentro del puerto pesquero; y, finalmente, cohesionar el trabajo de los Gremios de la provincia por la proximidad de los elementos mantenidos en un ambiente de tranquilidad y cultura edificante y progresista. (Instituto de Fomento Pesquero de Tarapacá, 1936, p. 667)

Lo anterior nos remite al concepto de seguridad social como gestión dentro de la racionalidad modernizadora o capitalista, la cual buscaba la normalización y homogenización de la población de pescadores como regulación que se expresaba hacer vivir y dejar morir (Foucault, 2001). Tales discursos ideológicos operaban como verdades absolutas de los conceptos 
de desarrollo y progreso de la época, los cuales se tornaron necesarios para generar cambios en los hábitos locales, reemplazándolos por otros más adecuados al nuevo modelo productivo, legitimados según criterios de utilidad y eficiencia (Aguirre et al., 2018).

Dentro de esta misma lógica se pueden visualizar los relatos que expresaban la problemática de la "raza" de los pescadores, concepto utilizado para manifestar el obstáculo que generaría cierta condición biológica y cultural de estas poblaciones para la consecución de los procesos de desarrollo industrial que se quería implementar. A modo de ejemplo, tenemos la siguiente cita:

Se desea aumentar en forma apreciable el elemento pescador de la provincia, en tal forma que represente una garantía de trabajo para esta industria. Para llenar esta finalidad es necesario cambiar radicalmente la idiosincracia del pescador regional mezclándolo con elemento extranjero, esforzado en la faena pesquera y habituado a métodos más modernos de pesca (...). (Instituto de Fomento Pesquero de Tarapacá, 1936, p. 1315)

De igual forma se pone énfasis en la precariedad tecnológica y técnica del pescador, las cuales son vistas como atrasadas y obsoletas, asumiendo que el conocimiento local no se ajustaba a los requerimientos y aspiraciones de desarrollo económico y cultural de la época. De acuerdo con ello se manifestaba:

(...) que se debe buscar en Europa, y en especial en los países escandinavos, hombre que haya tenido gran experiencia en la dirección de una fábrica. Nos hace conveniencia de traer un hombre europeo de reconocida competencia, junto con unas seis familias de pescadores de alta mar. (Plan Pesquero, 1936, p. 12)

Esto nos recuerda el clásico binomio conceptual de la construcción del otro que opone a civilizados/incivilizados, desarrollados/subdesarrollados, además de lo útil/inútil (Aguirre et al., 2018).

En este contexto, los dispositivos disciplinarios se tornaron necesarios para generar cambios en los hábitos culturales locales, reemplazándolos por otros más acordes al nuevo modelo productivo, modelo que se constituye en la separación de la fuerza de trabajo entre el centro y la periferia (Wallerstein, 2003). Con esto se puede sostener que este proyecto industrializador y sus dispositivos apuntaban a disciplinar y construir cuerpos productivos dóciles y útiles al nuevo sistema que se quería imponer y, a la vez, favorecer la valorización económica de la vida misma de las poblaciones pesqueras en el norte de Chile. 


\section{COMENTARIOS FINALES}

Hemos analizado el primer Plan Pesquero desarrollado por agencias del Estado en las costas del norte de Chile entre 1935 y 1938, proyecto que nace en un contexto de emergencia económica derivada del cierre progresivo de las oficinas salitreras de la Región de Tarapacá y de la crisis internacional generada por la depresión de finales de la década de los años veinte del siglo pasado. La cesantía de miles de trabajadores vinculados a esa industria y sus actividades económicas asociadas motivaron la migración forzada hacia Iquique y otras ciudades costeras y posteriormente hacia Valparaíso, Santiago y Chile Central. Por lo tanto, era urgente plantear nuevas fuentes de trabajo y también reorientar la economía nacional. El modelo exportador tradicional entró en crisis y las rentas nacionales cayeron en forma vertiginosa, lo que también motivó conflictos sociales y políticos que se venían gestando desde antes. Estos procesos también se estaban dando en otros países de América y Europa. El capitalismo industrial y mercantil de las grandes potencias europeas dependientes del modelo exportador de materias primas de sus colonias o asociados se fracturó y provocó la miseria de millones de personas abriendo las puertas de la inestabilidad política, la violencia y la guerra en pocos años.

Desde 1920 se aprecian en Chile una serie de procesos y cambios motivados tanto por la situación internacional como nacional: la crisis terminal del sistema parlamentario y la riqueza salitrera que lo sostenía, las migraciones rurales hacia las urbes en la búsqueda de mejores oportunidades y el crecimiento no planificado de las ciudades, la emergencia de las clases medias urbanas, la consolidación de los movimientos obreros y la formación de nuevos partidos políticos. Lo anterior, junto a otras situaciones, motivó que se impulsaran en Chile cambios profundos en el contexto de una inestabilidad política aguda que llevaron a sucesivos gobiernos y golpes militares. El sistema político debió cambiar y también se debía diseñar un nuevo sistema económico. La ideología que se fue imponiendo fue la de la industrialización del país, la sustitución creciente de las importaciones y la explotación de nuevos recursos naturales. En este contexto el extenso mar chileno y sus pesquerías se presentaron como una gran oportunidad, siendo el norte de Chile con su riqueza marina no explotada industrialmente un aliciente para ello.

El Plan Pesquero ideado en 1935 tenía como objetivo central crear la pesquería industrial en el norte de Chile. Para ello se crearon instituciones públicas de fomento para el sector, tanto en Antofagasta como en Iquique, las cuales tuvieron a su cargo la planificación inicial, la organización de los 
pescadores, estimular la participación del sector privado, la inversión básica en equipos y embarcaciones, facilitar la comercialización del pescado, la propaganda para su consumo por parte de la población y la capacitación de la fuerza de trabajo para mejorar su productividad.

Esta primera fase de industrialización tuvo un impacto favorable inicial, pero debió reconocer que su crecimiento estaba limitado por las características propias de la pesca en el medio marino, el poco interés de los capitales privados por invertir en un negocio nuevo e inestable en sus rentas, además de la necesidad de cambiar ciertas pautas culturales y sociales de los pescadores que no se ajustaban al modelo industrial que se quería implementar. Esto último llevó a plantear la necesidad de traer mano de obra especializada del extranjero y disciplinar a los pescadores con nuevas normativas legales sobre su actividad y asentamientos.

Finalmente, el Plan Pesquero debió reconocer en la práctica que no bastaba con fomentar la actividad y ordenar el sector. Si bien lo anterior era una condición necesaria, sería fundamental que el Estado tuviera un rol clave en la inversión de capitales y en la instalación y operación de las primeras fábricas de conservas y derivados de la producción pesquera, por lo menos hasta que el sector privado viera que su participación financiera fuera segura y el Estado diera garantías de un ordenamiento de la pesquería industrial y sus trabajadores. Estos procesos son claves para comprender la complejidad del fenómeno industrializador y los modelos de acumulación, en los espacios marinos nortinos, aportando antecedentes relevantes como base para entender los procesos extractivistas implementados en décadas posteriores, y sus efectos asociados, en los territorios y poblaciones costeras nortinas.

\section{REFERENCIAS}

Aguirre, C. (2011). Imaginarios sociales en el puerto de Iquique en la memoria de los pescadores industriales (1960-1990). Tesis para a optar al grado de Magíster en Antropología. Universidad Católica del Norte, Antofagasta, Chile.

Aguirre, C. (2016). Capitalismo y pesca industrial en el norte de Chile. Relaciones de producción, género y sociabilidad en el puerto de Iquique durante el ciclo pesquero industrial (1950-1990). Tesis para optar grado de Doctor en Antropología. Universidad Católica del Norte, Antofagasta, Chile.

Aguirre, C., Díaz, A. y Mondaca, C. (2014). De pescador artesanal a tripulante pesquero. Pesca industrial y transformacionales sociales en la Región de Tarapacá (1950-1990). Intersecciones en Antropología 1, 177-185. 
Aguirre, C., Mondaca, C. y Muñoz, W. (2018). Desarrollismo y capitalismo en espacios marinos. La industrialización de la pesca en Iquique, Norte de Chile. Interciencia 45(9), 611-618.

Albert, F. (1913). El problema pesquero en Chile. Santiago: Kosmos.

Alegret, J. (1988). Corporativismo, Intervención Estatal y Transformación Capitalista en el Sector Haliéutico: Las Cofradías de Pescadores de Cataluña. Tesis para optar al grado de Doctor en Antropología, Universidad Laval, Quebec.

Alfaro, C. (1936). Reseña histórica de la Provincia de Tarapacá. Iquique: Imprenta Caras y Caretas.

Bolster, W. J. (2006). Opportunities in Marine Environmental History. Environmental History 11(3), 567-597.

Breton, Y. y López, E. (1989). Ciencias sociales y desarrollo de las pesquerías. Modelos y métodos aplicados al caso de México. Ciudad de México: Instituto Nacional de Antropología.

Camus, P., Hidalgo, R. y Muñoz, J. (2016). Las disputas por el mar: bienes comunes, pescadores artesanales y pesca industrial. El caso de la caleta Cocholgüe en el litoral centro sur de Chile a mediados del siglo XX. Población \& Sociedad 2, 91-114.

CORFO (1967). Geografía económica de Chile. Santiago: Corporación de Fomento de la Producción.

Durrenberger, E. \& Pálsson, G. (1987). Ownership at Sea: Fishing Territories and Access to Sea Resources. American Ethnologist 14(3), 508-522.

Foucault, M. (2001). Defender la sociedad. Buenos Aires: Fondo de Cultura Económica.

Harms, C. (1930). Los grandes problemas de la zona norte de Chile. Santiago: La Ilustración.

Lander, E. (2002). La utopía del mercado total y el poder imperial. Revista Venezolana de Economía y Ciencias Sociales 2, 51-79.

McCay, B. (1978). Systems Ecology, People Ecology, and the Anthropology of Fishing Communities. Human Ecology 6(4), 397-492.

Mendoza, G. (1962). La situación económica y social del gremio de los pescadores de Chile. Memorial del Ejército de Chile 310, 82-102.

Nazer, R. (2009). Historia de la Corporación de Fomento de la Producción CORFO 1939-2009. Santiago: Patrimonio Consultores.

Orrego, F. (1973). Chile y el derecho del mar. Santiago: Andrés Bello.

Oya, J. (1974). Las pesquerías españolas en onda larga: la gran pesca del bacalao en los bancos del Atlántico noroccidental. Geografía 10, 48-78.

Instituto de Fomento Pesquero de Tarapacá (1936). Plan pequero de Tarapacá. Iquique, Chile: Instituto de Fomento de Tarapacá.

Wallerstein, I. (2003). El moderno sistema mundial I. La agricultura capitalista $y$ los orígenes de la economía-mundo europea en el siglo XVI, décima edición. México D.F.: Siglo XXI. 